\title{
STREPTOCOCCUS AGALACTIAE EN GESTANTES DE 35 A 37 SEMANAS QUE ACUDEN A CONTROL PRENATAL EN EL INSTITUTO NACIONAL MATERNO PERINATAL
}

\author{
Elmer Jhony Nauto Ccorihuaman ${ }^{1}$
}

\begin{abstract}
RESUMEN
Objetivo: Determinar los niveles de colonización de Streptococcus agalactiae en secreciones vaginales y ano rectales de mujeres embarazadas de 35 a 37 semanas de gestación entre los meses de Febrero a Julio del 2017. Material y métodos: Se realizó un estudio de tipo descriptivo, prospectivo de corte transversal. Usando un muestreo no probabilístico por conveniencia se captaron 130 gestantes durante su control prenatal en el Instituto Nacional Materno Perinatal; mediante hisopado se obtuvieron muestras de secreción vaginal y ano rectal empleando como medio de enriquecimiento selectivo caldo Todd Hewitt suplementado con gentamicina $(0.8 \mathrm{mg} / \mathrm{mL})$ y ácido nalidíxico $(15 \mathrm{mg} / \mathrm{ml})$. El cultivo se realizó en agar sangre de carnero al 5\%, identificándose el germen mediante el tipo de hemólisis. Resultados: Se aislo Streptococcus agalactiae en 30 pacientes $(23,1 \%)$. Tanto las variables tiempo de gestación, edad materna ni número de partos se asociaron con la presencia de Streptococcus agalactiae. Conclusiones: La prevalencia de la colonización vaginal y ano rectal por Streptococcus agalactiae en nuestro hospital es superior a la esperada en América Latina y se asocia a la edad materna, numero de gestación y nivel de instruccion.
\end{abstract}

Palabras claves: Streptococcus agalactiae, recuento de colonia microbiana, frotis vaginal, control prenatal (Fuente: DeCS BIREME).

\section{STREPTOCOCCUS AGALACTIAE IN PEOPLE FROM 35 TO 37 WEEKS GOING TO PRENATAL CONTROL IN THE NATIONAL PERINATAL MATERNAL INSTITUTE}

\begin{abstract}
Objective: Determine the level of colonization in vaginal smears in 35 to 37 pregnant women during February to July of 2017. Material and methods: A descriptive, prospective, transversal study was design. Using a non probabilistic sampling for convenience, we selected 130 pregnant women during their prenatal care in the perinatal maternal national institute. We obtained samples of vaginal and rectal anal smears by swab using a Todd Hewitt medium supplemented with Gentamicin $(0.8$ $\mathrm{mg} / \mathrm{ml})$ and nalidixic acid $(15 \mathrm{mg} / \mathrm{ml})$ as a selective enrichment medium. The culture was carried out in $5 \%$ ram blood agar, and the gem was identified by the type of hemolysis. Results: Streptococcus agalactiae was isolated in 30 patients $(23.1 \%)$. Time of gestation, maternal age or number of deliveries was asociated with the presence of Streptococcus agalactiae in the culture. Conclusions: the prevalence of the vaginal rectal anal colonization of Streptococcus agalactiae is higher than the prevalence in Latin America, it is associated with the age of the mother, the number of gestations and the instruction level of the mother.
\end{abstract}

Key words: Streptococcus agalactiae, colony count, microbial, Vaginal smears, prenatal care (Source: MeSH NLM).

\section{INTRODUCCIÓN}

El cuerpo humano es un complejo ecosistema formado por $10 \%$ células humanas y $90 \%$ de microorganismos. Estos dos componentes trabajan de forma integrada para permitir el funcionamiento de los sistemas del cuerpo ${ }^{1}$. El Streptococcus agalactiae (conocido también como Estreptotococo Beta Hemolítico Grupo B o GBS por sus siglas en inglés) es una bacteria que puede habitar el trato genital femenino y el recto, con tasas de colonización que oscilan entre el 30\% (Jordania) y $2.9 \%$ (Japón) ${ }^{2,3}$.

Según la literatura internacional, alrededor del $20 \%$ (con un rango de 10 a $40 \%$ ) de las mujeres embarazadas son portadoras de este germen en vagina y /o recto al final del embarazo, con mayor frecuencia en la raza negra 4,5 . Algunos estudios en Argentina muestran una incidencia más baja: del 5 al $18 \%{ }^{6}$. Cuando existe colonización materna, si no se efectúa ninguna medida de prevención, $50-70 \%$ de los neonatos se colonizan durante el parto, pero sólo $1-2 \%$ de ellos se enferma ${ }^{7}$.

El Instituto Nacional Materno Perinatal tiene como visión dar atención especializada y altamente especializada a la mujer en salud Sexual y reproductiva y al neonato de alto riesgo, desarrollando investigación, tecnología y docencia a nivel nacional, por lo que no teniendo datos propios de la institución acerca del estado actual de estas condiciones

\footnotetext{
Lic. Tecnólogo Médico con especialidad de laboratorio clínico y anatomía patológica

Citar como: Nauto EJ. Streptococcus agalactiae en gestantes de 35 a 37 semanas que acuden a control prenatal en el instituto nacional materno perinatal. Rev Peru Investig Matern Perinat 2019; 8(4): 25-9

DOI https://doi.org/10.33421/inmp.2019170
} 
en las mujeres que acuden a control prenatal es que dicho hospital ha mostrado interés en que el siguiente protocolo se realice con su población.

\section{MATERIALES Y MÉTODOS}

La investigación es de tipo descriptivo, prospectivo, de corte transversal. Se tomó como población a todas las gestantes de 35 a 37 semanas que acuden al control prenatal en el área de obstetricia del Instituto Nacional Materno Perinatal en Lima, Perú; durante el periodo de Febrero a Julio del 2016 aplicando un muestreo no probabilístico por conveniencia. Dentro de los criterios de inclusión se eligieron a las pacientes embarazadas con 35 a 37 semanas de gestación establecidas por última fecha de menstruación (FUM) o ecografía temprana; de cualquier edad y paridad; que otorguen su consentimiento informado; que no hayan recibido tratamiento con antibióticos por lo menos 15 días antes del estudio; que no hayan recibido tratamiento local vaginal con antimicrobianos por lo menos 48 horas antes del estudio. No se procesaron aquellas muestras que no hayan sido conservadas adecuadamente ni se consideraron a las pacientes que presentaron enfermedades genitales víricas y/o micóticas en el momento del estudio. Para el análisis estadístico univariado se emplearon tablas de frecuencia absoluta y relativa mientras que para el análisis inferencial se utilizó chi cuadrado por medio del paquete estadístico SPSS v 23.0.

\section{Toma y procesamiento de la muestra}

A cada paciente se le solicitó su consentimiento por escrito para poder incluirle en el estudio previa entrevista en la cual se le informó previamente sobre los objetivos de la investigación, como sería manejada la información y, sobre los posibles riesgos de la toma de muestra. Además se le orientó y explicó la importancia de las infecciones causadas por la colonización de streptococcus agalactiae, el significado de un resultado positivo y un negativo. Se les realizo un hisopado de secreción vaginal y de zona ano rectal de las pacientes entre 35 y 37 semanas de gestación durante la consulta de control perinatal, la cual fue colocada en el medio de transporte de Amies para preservarla hasta su procesamiento y su posterior coloracion con tinción Gram. El hisopo del medio de transporte Amies se inoculo en un tubo con Caldo Todd Hewitt suplementado con gentamicina $(8 \mu \mathrm{g} / \mathrm{mL})$ y se incubó de $18-24$ horas a $37^{\circ} \mathrm{C}$ en la estufa. Al día siguiente con la ayuda del hisopo antes mencionado y de un asa bacteriológica estéril se procedió a realizar la siembra en agar sangre de cordero al $5 \%$ mediante la técnica de estría punción para el aislamiento de estreptococos $\beta$-hemolíticos y se incubaron las placas Petri a $37{ }^{\circ} \mathrm{C}$ por 24-48 horas. Todas las colonias que presenten una beta hemolisis estrecha se utilizaron para efectuar las pruebas de CAMP.

\section{RESULTADOS}

De las 130 gestantes estudiadas el promedio y desviación estándar de las edades fue 29,9 $\pm 5,9$ años, con una mediana de 29 años, una moda de 25 años y un rango entre 20 a 42 años. Del total de gestantes un $53,8 \%$ fue del grupo de edades entre 20 a 30 años y un $46,2 \%$ fue de las edades entre 31 a 42 años (Tabla1).

En relación al número de gestas, el promedio fue 2,4 \pm 1,1 , con una mediana y moda de 2 y un rango de 1 a 7 gestaciones. Del total de gestantes un $60 \%$ estuvo en el grupo de 1 a 2 gestas, un $34,6 \%$ entre 3 a 4 gestas y un $5.4 \%$ entre 5 a 7 gestas (Tabla 1).

En cuanto al grado de instrucción, un $18,5 \%$ tenía sólo educación primaria, un $54,6 \%$ tenía solo educación secundaria, $26,9 \%$ tenía educación superior, siendo las gestantes de educación secundaria, el grupo más representativo (Tabla 1 ).

Tabla 1. Características clínico demográficas

\begin{tabular}{|c|c|c|c|}
\hline \multicolumn{2}{|c|}{ Características } & \multirow{2}{*}{$\begin{array}{c}\mathbf{n} \\
70\end{array}$} & \multirow{2}{*}{$\begin{array}{c}\% \\
53.8\end{array}$} \\
\hline \multirow{3}{*}{ Edad } & 20-30 años & & \\
\hline & $31-42$ años & 60 & 46.2 \\
\hline & Total & 130 & 100 \\
\hline \multirow{6}{*}{ Gesta } & 01-feb & 78 & 60 \\
\hline & 03-abr & 45 & 34.6 \\
\hline & 05-jul & 7 & 5.4 \\
\hline & Total & 130 & 100 \\
\hline & Primaria & 24 & 18.5 \\
\hline & Secundaria & 71 & 54.6 \\
\hline \multirow[t]{3}{*}{ Grado de instrucción } & Superior técnico & 26 & 20 \\
\hline & Superior universitario & 9 & 6.9 \\
\hline & Total & 130 & 100 \\
\hline
\end{tabular}

Fuente: elaboración propia

Se encontraron 30 casos positivos para Streptococcus agalactiae de un total de 130 gestantes entre las 35 a 37 semanas de gestación, quienes acudieron a control prenatal en el Instituto Nacional Materno Perinatal, lo cual representa una prevalencia de $23,1 \%$ (Tabla 2 ). 
Tabla 2. Frecuencia de Streptococcus agalactiae en mujeres embarazadas de 35 a 37 semanas de gestación.

\begin{tabular}{lcc}
\hline & N & \% \\
\hline Positivo & 30 & 23.1 \\
Negativo & 100 & 76.9 \\
Total & 130 & 100 \\
\hline
\end{tabular}

Fuente: elaboración propia

Para la asociación de número de gestas y colonización de Streptococcus agalactiae, el grupo más representativo tuvieron entre 3 a 4 gestas $(70,0 \%)$, seguido de las gestantes que tuvieron entre 5 a 7 gestas $(16,7 \%)$ y las gestantes que tuvieron entre 1 a 2 gestas (13,3\%) (Tabla 3). Se encontró asociación estadísticamente significativa entre el número de gestas y la colonización de Streptococcus agalactiae $(p<0,001)$.

Tabla 3. Características clínico demográficas según presencia de Streptococcus agalactiae

\begin{tabular}{|c|c|c|c|c|c|}
\hline & \multicolumn{4}{|c|}{ Streptococcus agalactiae } & \multirow[b]{3}{*}{$\mathbf{p}$} \\
\hline & \multicolumn{2}{|c|}{ Positivo } & \multicolumn{2}{|c|}{ Negativo } & \\
\hline & $\mathbf{n}$ & $\%$ & $\mathbf{n}$ & $\%$ & \\
\hline \multicolumn{6}{|c|}{ Edad } \\
\hline 20-30 años & 10 & 33.3 & 60 & 60 & \\
\hline 31-42 años & 20 & 66.7 & 40 & 40 & 0.01 \\
\hline Total & 30 & 100 & 100 & 100 & \\
\hline \multicolumn{6}{|c|}{ Numero de gestas } \\
\hline $1-2$ & 4 & 13.3 & 74 & 74 & \\
\hline $3-4$ & 21 & 70 & 24 & 24 & $<0.0001$ \\
\hline $5-7$ & 5 & 16.7 & 2 & 2 & \\
\hline \multicolumn{6}{|c|}{ Grado de instrucción } \\
\hline Primaria & 12 & 40 & 12 & 18.5 & \\
\hline Secundaria & 13 & 43.3 & 58 & 54.6 & 0.003 \\
\hline Superior técnico & 5 & 16.7 & 30 & 26.9 & \\
\hline
\end{tabular}

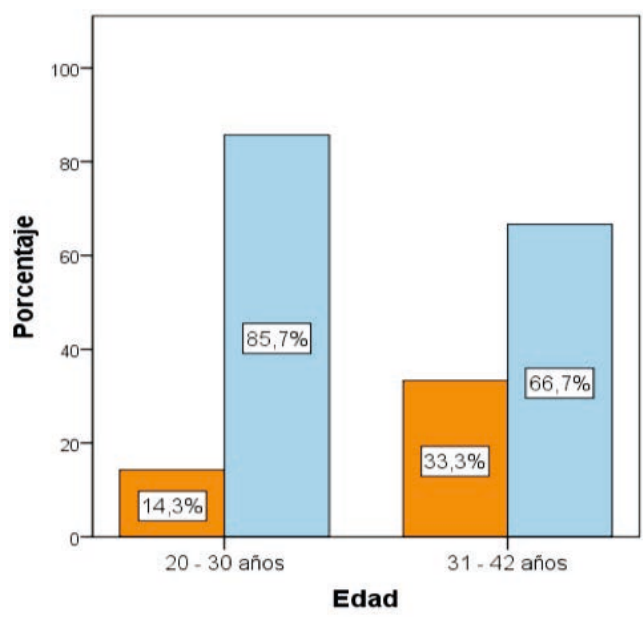

Gráfico 1. Frecuencia de gestantes con Streptococcus agalactiae según la edad.

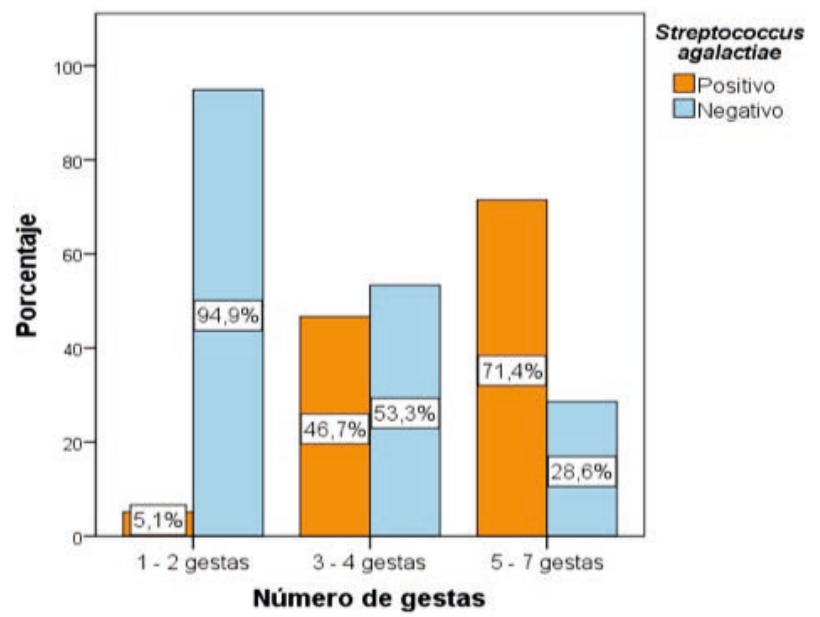

Gráfico 2. Frecuencia de gestantes con Streptococcus agalactiae según el número de gestas. 
En cuanto al grado de instrucción de las 30 gestantes positivas a Streptococcus agalactiae, 12 (40,0\%) tuvieron sólo educación primaria, $13(43,3 \%)$ tuvieron educación secundaria y $5(16,7 \%)$ gestantes tuvieron educación superior. Ninguna gestante tuvo educación superior universitaria (Tabla 3 ). Se encontró asociación estadísticamente significativa entre grado de instrucción y colonización de Streptococcus agalactiae $(p=0,002)$.

\section{DISCUSIÓN}

Durante el período Febrero a Julio de 2017, se procesaron muestras de secreción vaginal e hisopado ano rectal de 130 mujeres embarazadas entre 35 y 37 semanas de gestación que acudieron a la consulta prenatal al Instituto Nacional Materno Perinatal, con el objetivo de conocer la prevalencia de colonización de streptococcus agalactiae. Las muestras de las pacientes se obtuvieron según los parámetros indicados por el instituto nacional de salud, las cuales además debían cumplir con los criterios de inclusión y exclusión establecidos para el estudio. Las cepas recuperadas de streptococcus agalactiae. Correspondieron al $23.1 \%$ de las muestras analizadas considerando el cultivo de secreción vaginal así como el de hisopado ano rectal, porcentaje mayor del rango obtenido en otras poblaciones de América que oscila entre el 10 al $20 \%$, según Casal et al ${ }^{8}$.

En Chiapas-México en el año 2013 por Ocampo et al, 9 encontraron una prevalencia del $8,6 \%$ diferente al realizado en Venezuela en el año 2015 por Armas con una prevalencia del $21.1 \% \%$ el cual se acerca mas a nuestros resultados. Comparando con investigaciones realizadas entre las 35 y 37 semanas de gestación nuestro trabajo tiene resultados superiores al obtenido en otros estudios estudios latinoamericanos como los llevados a cabo en Brasil (2013) por Veit et al, y en Chile (2012) por Valdés et al ${ }^{10}$, con prevalencias del $11,11 \%$ y $14 \%$ respectivamente.

La prevalencia podría variar en diferentes lugares de Latinoamérica dependiendo del tipo de estudio, del medio de cultivo utilizado y del tipo de población tamizada, con o sin factores de riesgo. Así en otros artículos publicados encontramos resultados diferentes, como los obtenidos en México (2019) por Campo et al, con el $20.66 \%$ en Perú ${ }^{11}(2004)$ por Tamariz et al, con el $10,9 \%$; en Nicaragua (2014) por Dubón et al, con el $11 \%{ }^{12}$ y en Guatemala (2003) por Pereira con el $14,4 \%{ }^{13}$.

En conclusión con los resultados obtenidos en la presente investigación: la prevalencia de Streptococcus agalactiae en gestantes de 35 a 37 semanas que acuden a control prenatal en el Instituto Nacional Materno Perinatal es mayor (23.1\%) de la planteada de $20 \%$ a nivel internacional. Además se encontró asociación entre la edad, numero de gestaciones y grado de instrucción; y la colonización por Streptococcus agalactiae.

\section{RECOMENDACIONES}

- Implementar el cultivo rutinario para detección de Streptococcus agalactiae en pacientes embarazadas de 35 a 37 semanas de gestación y dar seguimiento a las gestantes portadoras antes del parto con el fin de prevenir complicaciones posteriores en el recién nacido.

- Ampliar estudios que involucren el empleo de medios selectivos como el caldo Todd Hewitt que favorecen la recuperación de Streptococcus agalactiae así como de medios de transporte (Stuart) que mantienen viables los microorganismos hasta el momento de su procesamiento, para determinar su viabilidad y su posible implementación en el laboratorio de esta institución.

\section{AGRADECIMIENTOS}

A los médicos de consulta externa como Dr. Villanueva. Dr, Loarte. Dr Montes, Dr Miranda, Dr Chinchayan por su apoyo y confianza en nuestro trabajo. Gracias por su paciencia y sus consejos que de seguro nos serán muy útiles toda la vida. Expreso también mi agradecimiento al Dr. Enrique Guevara Ríos Director del Instituto Nacional Materno Perinatal por permitirnos desarrollar este trabajo de investigación.

\section{REFERENCIAS BIBLIOGRÁFICAS}

1. P. Murray, Pfaller M. Microbiologia Medica. 5ta Ed. Elsevier; 2016. p. 3, 12, 13.

2. Luna Sanchez Hector Jose. Estreptococo Beta Hemolítico del Grupo B en Pacientes con Trabajo de Parto Pretermino y su Relación con Corioamnionitis Subclínica y Sepsis Neonatal. [Guatemala]: Universidad Francisco Marroquin; 2014.

3. BARTOLOMEO S, GENTILE M, PRIORE G, VALLE S, BELLA A. Streptococcus agalactiae en embarazadas. Prevalencia en el Hospital Nacional Alejandro Posadas. Rev Argent Microbiol. 1 de septiembre de 2005;37.

4. Whitnack E. Estreptococos. En: Microbiologia: mecanismo das doencas infecciosas. Schaechter. Brasil; 2012.

5. Rosa M, Prieto J. Microbiologia en Ciencias d e la Salud. 2da ed. España: Elsevier; 2016. p. 12, 13.

6. Centelles-Serrano MJ, Pérez-Moreno MO, LlovetLombarte MI, Cortell-Ortolá M, Jardí-Baiges AM, BujGonzález Jl. Impacto de la investigación sistemática de estreptococo del grupo B en orina en la identificación de gestantes colonizadas. Enfermedades Infecc Microbiol Clínica. 1 de agosto de 2009; DOI: 27(7):394-8. 10.1016/j. eimc.2008.10.004

7. Meis PJ, Goldenberg RL, Mercer B, Moawad A, Das A, McNellis $D$, et al. The preterm prediction study: significance of vaginal infections. National Institute of Child Health and Human Development Maternal-Fetal Medicine Units Network. Am J Obstet Gynecol. octubre de 1995;173(4):1231-5. DOI: 10.1016/0002-9378(95)91360-2

8. Ministerio de Sanidad servicios Sociales e igualdad. Guia de practica clinica de atencion en el embarazo y puerperio [Internet]. Agencia de Evaluacion de Tecnologias Sanitarias de Andalucia; 2014. Disponible en: https://portal.guiasalud. es/wp-content/uploads/2018/12/GPC_533_Embarazo_ AETSA_compl.pdf

9. Ocampo-Torres $M$, Sánchez-Pérez HJ, NazarBeutelspacher A, Castro-Ramírez AE, Cordero-Ocampo B. Factores asociados a la colonización por Streptococcus del grupo B en mujeres embarazadas de Los Altos, Chiapas. Salud Pública México. septiembre de 2000;42:413-21. DOI: 10.1590/S0036-36342000000500007

10. Valdés RE, Pastene SC, Morales PA, Gutiérrez RB, Canales $\mathrm{P} A$, Martínez $\mathrm{O} \mathrm{P}$, et al. Prevalencia de colonización por Streptococcus Agalactiae (GRUPO B) durante el embarazo 
pesquisado en medio de cultivo selectivo. Rev Chil Obstet Ginecol. 2004;69(2):132-5.

11. Campo Cesar Hernan, Martinez Maria Fernanda, Otero Juan Carlos, Rincon Giovanna. Prevalencia de colonizacion vagino rectal por Streptococcus agalactiae y su perfil de sensibilidad en mujeres embarazadas atendidas en un hospital de tercer nivel. Biomedica. 2019;39:689-98. DOI: $10.4067 /$ S0717-75262004000200008

12. Dubón Mendez N, Altamirano Gonzélez M del S, Aleman Rivera T de J. Streptococo del grupo B en mujeres embarazadas atendidas en el Centro de Salud Primero de Mayo. Abril-Agosto 2007 [Internet]. Universitas (León). Revista Científica de la UNAN-León. Vicerrectoria de Investigación, Postgrados y Proyección Social. 2008 [citado 10 de diciembre de 2019]. Disponible en: https:// ageconsearch.umn.edu/record/207407 DOI: DOI: 10.22004/ ag.econ.207407

13. CARMEN ESTHELA PEREIRA QUIÑONEZ. Detección de Streptococcus agalactiae en Mujeres Embarazadas que Acuden a la Consulta Prenatal del Hospital General San Juan de Dios. [Guatemala]: Universidad San Carlos de Guatemala;

\section{Correspondencia:}

Elmer Jhony Nauto Ccorihuaman

Dirección: Av. Canadá 668 Urb. Perú Zona San Martín de Porres

Correo:Jhonny_4414@hotmail.com

Teléfono: 989533168 